\title{
EFECTO EN LAS PROPIEDADES MECÁNICAS DE UNA RESINA PINIFERA MODIFICADA BIODEGRADABLE, AL UTILIZARLA COMO COMPATIBILIZANTE O ACOPLANTE EN FORMULACIONES ELASTOMERICAS SIN O CON FIBRA DE AGAVE Y HULE DE POLI (ESTIRENO-BUTADIENO). UN PASO HACIA LA FORMULACION DE ELASTOMEROS VERDES
}

\author{
EFFECT ON MECHANICAL PROPERTIES OF A BIODEGRADABLE \\ MODIFIED PINE RESIN, WHEN USING IT AS COMPATIBILIZER OR \\ COUPLING AGENT IN ELASTOMERIC FORMULATIONS WITHOUT \\ OR WITH AGAVE FIBER AND POLY(STYRENE-BUTADIENE) \\ RUBBER. ONE STEP TOWARDS GREEN ELASTOMERS \\ FORMULATION
}

\author{
Francisco José Aranda-García ${ }^{1}$ \\ https://orcid.org/0000-0002-3708-9761 \\ Mónica Paola Rodríguez-Ortiz ${ }^{2}$ \\ https://orcid.org/0000-0001-6947-4595 \\ Eduardo Mendizábal ${ }^{3}$ \\ http://orcid.org/0000-0003-4672-2052 \\ Carlos F. Jasso-Gastinel ${ }^{2, \$}$ \\ https://orcid.org/0000-0003-0522-0945
}

\begin{abstract}
RESUMEN
En este trabajo se valora la eficiencia de una resina de pino modificada biodegradable (A-30), al utilizarla como compatibilizante o acoplante para sustituir al resorcinol (que es tóxico y no es biodegradable), comparando las propiedades mecánicas (contra formulaciones preparadas usando el sistema resorcinol-hexametilentetramina (R-HMT) en: a) polímeros elastoméricos reticulados de estireno-butadieno (SBR), y: b) compósitos de SBR reforzados con fibra de agave de desecho. Para la comparación como compatibilizante, aquí se preparan formulaciones equivalentes con A-30 o R-HMT. Para la comparación de los compósitos equivalentes, se varía el agente de acoplamiento (R-HMT o A-30) y la cantidad de azufre como agente de entrecruzamiento (2,5 partes por cien de elastómero ( $\mathrm{phr}$ ), 7,5 phr o $15 \mathrm{phr}$ ). En las pruebas de tracción a temperatura ambiente, y el análisis mecanodinámico con barridos de temperatura $\left(\mathrm{de}-70{ }^{\circ} \mathrm{C}\right.$ a $\left.40{ }^{\circ} \mathrm{C}\right)$ y frecuencia $(\mathrm{de} 0,4 \mathrm{~Hz}$ a 40 $\mathrm{Hz}$ ), se obtuvieron incrementos en módulos (hasta un $74 \%$ en módulo de Young) y en capacidad de deformación (hasta $260 \%$ ), para los materiales conteniendo el agente A-30, por encima de las formulaciones en las que se usó el resorcinol. El mejor desempeño como compatibilizante o acoplante del A-30, se reflejó también con claridad morfológicamente mediante microscopía electrónica de barrido, al mostrar mayor compacidad en la matriz formulada y mayor cercanía entre fibra y matriz en muestras fracturadas criogénicamente. Se ha demostrado aquí, que el A-30 puede sustituir al par R-HMT con éxito, ya que funciona mejor como agente compatibilizante o acoplante y es biodegradable. Este tipo de agentes tiene un gran potencial para contribuir al
\end{abstract}

\footnotetext{
${ }^{1}$ Universidad de Guadalajara, Departamento de Ingeniería, CUCSUR. Autlán de Navarro, Jalisco, México

${ }^{2}$ Universidad de Guadalajara, Departamento de Ingeniería Química, CUCEI. Guadalajara, Jalisco, México.

${ }^{3}$ Universidad de Guadalajara, Departamento de Química, CUCEI. Guadalajara, Jalisco, México.

•Autor para correspondencia: carlos.jasso@cucei.udg.mx

Recibido: 16.11.2020 Aceptado: 20.07.2021
} 
desarrollo de elastómeros y compósitos flexibles amigables con el medio ambiente.

Palabras clave: Agente de acoplamiento, compatibilizante, compósito de SBR/fibra, fibra de agave, resina de pino, resina maleinizada.

\begin{abstract}
In this work, the effectiveness of a biodegradable modified pine rosin (Amberyl MP-30 (A-30)), is evaluated as compatibilizer or coupling agent to substitute resorcinol (which is toxic and non-biodegradable) in elastomeric formulations. Their mechanical properties are compared vs formulations containing resorcinol-hexamethylenetetramine (R-HMT) in: a) styrene-butadiene elastomers (SBRs), and: b) SBR composites reinforced with discarded agave fibers. For the comparison as compatibilizer, equivalent formulations containing resorcinol or A-30 are prepared. Likewise, for the equivalent composites comparison, the coupling agent (R-HMT or A-30) and sulfur amount as crosslinking agent (2,5 parts per 100 rubber (phr), 7,5 phr or 15 parts per $100 \mathrm{phr}$ ) are varied. The Tensile tests at room temperature, and the mechanodynamic analysis using sweeps of temperature (from $-70{ }^{\circ} \mathrm{C}$ to $40{ }^{\circ} \mathrm{C}$ ) and frequency (from $0,4 \mathrm{~Hz}$ to $40 \mathrm{~Hz}$ ), allowed the observation of moduli improvements (up to $74 \%$ in Young modulus) and deformation capacity (up to $260 \%$ ) for the materials with A-30 over the materials where resorcinol was used. The better performance as compatibilizer or coupling agent of the A-30 was also shown with clarity in the morphological characterization, showing higher compacity in the formulated polymer matrix, and higher closeness between matrix and fibers, comparing with fractured samples using R-HMT. It has been shown here, that formulating rubbers or elastomeric composites with A-30 and HMT, can successfully substitute the R-HMT pair, because it works better as compatibilizer or coupling agent and is biodegradable. Agents of natural origin like this one, have a great potential to contribute to the development of elastomers and flexible composites that are environmentally friendly.
\end{abstract}

Keywords: Coupling agent, compatibilize, SBR/fiber composite, agave fiber, pine resin, maleated rosin.

\title{
INTRODUCCIÓN
}

En la producción de artículos industriales, se tiene la necesidad de preparar y/o utilizar productos poliméricos naturales o sintéticos que posean propiedades tales como baja conductividad térmica y/o eléctrica, así como flexibilidad y alta elasticidad (Geethamma et al. 2005), para usarlos como mangueras, cojines, guantes, sellos, neumáticos, cintas transportadoras o montajes en piezas metálicas entre otros (Zhang et al. 2015, Zhou et al. 2015). Sin embargo, para la elaboración de productos elastoméricos o ahulados es necesario añadir varios aditivos para obtener mezclas homogéneas y reticulantes o vulcanizantes (generalmente azufre por su bajo costo) para impartir estabilidad térmica y elasticidad al producto. Las concentraciones de azufre varían de acuerdo a la elasticidad requerida; por ejemplo, se tienen reportes de hules con elevada elasticidad al utilizar una concentración de azufre de 2,5 phr (Varghese et al. 1994, López-Manchado y Arroyo 2002), 15 phr (Abd-El-Messieh y Abd-El-Nour 2003), o con $30 \mathrm{phr}$ cuando se requiere obtener productos rígidos como la ebonita (Ciesielski 1999). Además, para mejorar la resistencia a la abrasión, rigidez y resistencia a la intemperie a estos materiales normalmente se les adicionan materiales de refuerzo como el negro de humo o la sílice (Zhou et al. 2015).

Como agentes de refuerzo en cauchos naturales y sintéticos se ha reportado el uso de fibras lignocelulósicas (Jasso-Gastinel et al. 1992, Mohanty et al. 2001, López-Manchado y Arroyo 2002, Jacob et al. 2004, Parambath-Kanoth et al. 2019) las cuales promueven incrementos en los módulos de tracción, flexión y la estabilidad térmica del hule, ampliándose el rango de sus aplicaciones (Thomas et al. 2015, Tian et al. 2019). Entre las ventajas del uso de tales fibras, se tienen su abundancia, inocuidad, biodegradabilidad, baja densidad, bajo costo, y valores elevados de los módulos de Young y de la resistencia específica, además de ser renovables (Poletto 2017, Pulido-González et al. 2014, Thomas et al. 2015, Mohanty et al. 2001). Entre los usos reportados de los hules reforzados con fibras se encuentran aplicaciones automotrices, empaques, productos de construcción, muebles, bienes de consumo (Meissner y Rzymski 2013) y montajes en piezas metálicas entre otros (Shahril et al. 2017).

Una de las fibras naturales de gran potencial para hacer este tipo de materiales es la obtenida como desecho del proceso de elaboración del tequila (Agave tequilana Var. Azul), cuya producción sigue en aumento cada año 
(Consejo Regulador del Tequila 2019).

Por la incompatibilidad entre los grupos funcionales de los hules (v.g. SBR) y las fibras, surge la necesidad de usar un agente acoplante, ya que al haber baja adherencia entre ellos, se presenta una separación de fases, lo cual conduce a una fuerte disminución de resistencia a la tracción última, además de la elongación al rompimiento (Liu et al. 2014, Pantamanatsopa et al. 2014, Lin et al. 2016). De hecho, en los materiales compuestos existen zonas donde las cadenas poliméricas del elastómero se encuentran inmovilizadas por los materiales de carga, conociéndose a esta zona como región restringida. Para reducir el área de la zona restringida, se han propuesto tratamientos físicos o químicos de la superficie de las fibras; el tratamiento químico ha demostrado ser útil generalmente para mejorar las propiedades mecánicas para módulo o deformación (Raza et al. 2016); sin embargo, pueden ser procesos lentos y encarecer los productos. Por otro lado, la adición de agentes de acoplamiento es un proceso más simple, como es el caso del R-HMT (Geethamma et al. 1995, Soltani et al. 2014).

En ausencia de carga reforzante, entre los aditivos se utiliza un compatibilizante para obtener mezclas homogéneas y el reticulante que determina la elasticidad y con ello las posibles aplicaciones del elastómero. Los compatibilizantes permiten una mejor interacción entre los componentes del sistema de vulcanización y los acoplantes lo hacen entre el elastómero formulado y el agente de refuerzo, para los compósitos. Sin embargo, los compuestos que se utilizan actualmente como compatibilizantes o acoplantes son contaminantes debido a su difícil degradación y recuperación (por ejemplo, el resorcinol) (Flickinger 1976, Tamura et al. 2012).

El uso del resorcinol en conjunto con otros agentes es muy utilizado para mejorar la adhesión de materiales de refuerzo, ya sean de tipo orgánico o inorgánico en la formulación de hules sintéticos o naturales. Como material compatibilizante el resorcinol tradicionalmente se ha usado en conjunto con formaldehído como látex ((RFL), Seo 1997, Debasish et al. 2006, Parambath-Kanoth et al. 2019) o como en este trabajo con hexametilentetramina ((R-HMT), Dressler 1994, Varghese et al. 1994, Jacob et al. 2006, El-Wakil 2011, Halim et al. 2013, Jiang y Gu 2020), donde se forma un complejo similar al que se forma con el RFL, el cual genera adhesividad (Lawandy et al. 2018, Wisojodharmo et al. 2020).

Ante tal panorama, en este trabajo se propone el utilizar la resina de pino maleinizada-(Amberyl MP-30 (A-30)), como compatibilizante en la formulación del SBR y por otra parte como acoplante entre el SBR y la fibra de agave. La resina de pino también conocida como colofonia, brea o goma rosin, es una mezcla de entre $90 \%$ y $95 \%$ resina de ácido abiético y ácido pimárico; tiene fórmula molecular de $\mathrm{C}_{20} \mathrm{H}_{30} \mathrm{O}_{2}$ y un peso molecular de $302 \mathrm{~g} / \mathrm{mol}$. Es un material sólido cristalino, soluble en solventes orgánicos; gracias a que no es soluble en agua impide la adsorción de ésta, y por otra parte, al no afectar la salud humana (Gallo-Corredor y Sarria-Villa 2013, Quiroz-Carranza y Magaña-Alejandro 2015) se le han encontrado aplicaciones en la industria farmacéutica. En su estructura química contiene un grupo carboxílico, varios anillos cíclicos y dobles enlaces conjugados, los cuales son susceptibles a modificaciones químicas (Sousa et al. 2019). La resina pinífera maleinizada se ha utilizado como agente de acoplamiento biodegradable en compósitos de termoplásticos/ fibra de agave (Pulido-González et al. 2014, Inga-Lafebre et al. 2019), o también como compatibilizante entre mezclas de polímeros biodegradables (Inga-Lafebre et al. 2019). En tal trabajo, la biodegradabilidad de la mezcla poli(ácido láctico) (PLA) y poli(tereftalato-co-adipato de butileno) (PBAT) fue comprobada tanto con brea gliceromaleica (Amberyl MP-15) como pentamaleica (Amberyl MP-30).

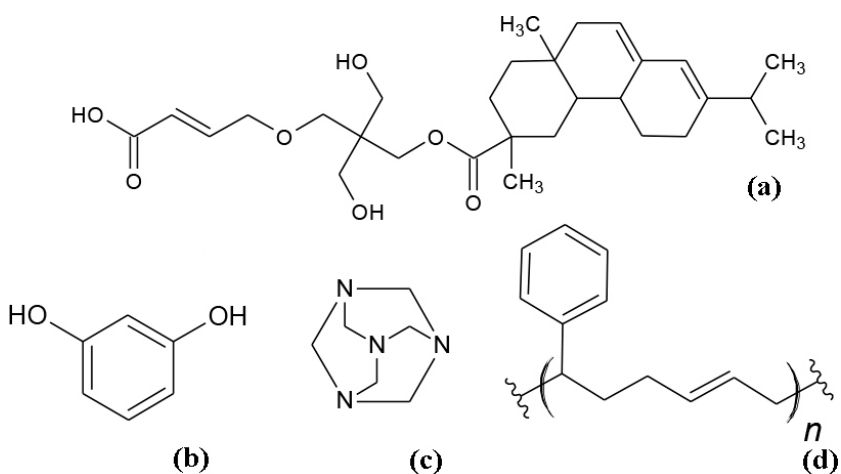

Figura 1: Estructuras químicas de los principales componentes para preparar los elastómeros/compósitos: (a) resina de pino pentamaleica, (b) Resorcinol, (c) Hexametilentetramina, (d) Elastómero SBR. 
Para la comparación entre los diferentes tipos de agentes comptibilizantes y acoplantes (Figura 1a, Figura $1 \mathrm{~b}$ and Figura 1c), en este trabajo se confrontan las propiedades mecano-estáticas (tracción) y mecanodinámicas (barridos de temperatura y frecuencia), en conjunto con la morfología (microscopía electrónica de barrido) que se obtiene utilizando la resina pentamaleíca, con las características obtenidas cuando se utiliza como compatibilizante o acoplante el par R-HMT.

Cabe destacar, que aunque en la literatura aparecen algunos trabajos de SBR (Figura 1d) reforzado con fibras lignocelulósicas, éste es el primer trabajo en el que se presenta el uso de un agente compatibilizante o acoplante de origen natural modificado biodegradable, lo cual representa una contribución al campo de los elastómeros y compósitos elastoméricos, a la vez que como agente de refuerzo se utiliza un material lignocelulósico económico de producción sostenible, por lo que se pretende mostrar que la producción de elastómeros y compósitos elastoméricos más amigables es factible, y muestran potencial para su uso en diversas aplicaciones industriales.

\section{MATERIALES Y MÉTODOS}

\section{Materiales}

El agente Amberyl A-30 (resina de pino pentamaleica) aquí utilizado, tiene número de acidez de 15 - 25 $\mathrm{mg} \mathrm{KOH} / \mathrm{g}$ y peso molecular de $504 \mathrm{~g} / \mathrm{mol}$ (proporcionado por Polímeros Sintéticos S.A. (MEXICO)), y es usado como compatibilizante o acoplante. El agente de referencia utilizado como compatibilizante y acoplante fue el resorcinol con pureza superior al 98,0 \% (Sigma -Aldrich) mezclado con la Hexametilentetramina (Hexa) pureza mayor al $97 \%$ (Sigma-Aldrich). El Hexa también participa como acelerador en conjunto con el 2-Mercaptobenzotiazol (MBT)(Aldrich), teniendo pureza mayor al $99 \%$. Como agente de refuerzo celulósico se utilizó fibra de bagazo de agave con una longitud promedio de $6,24 \mathrm{~mm} \pm 2,01 \mathrm{~mm}$ y una relación L/D promedio de 21,56, la cual provino como desecho de la industria tequilera de Tequila, Jalisco (México). Como matriz elastomérica se utilizó SBR (Solprene 1507), suministrado por Esponjas ABI S.A. de C.V., Guadalajara México (Figura 1d). Para el sistema de curado se utilizaron como activadores el óxido de zinc al 97,0 \% (Aldrich) y ácido esteárico con pureza superior al 95,0 \% (Sigma-Aldrich); este último también funcionó como agente dispersante. El azufre con 97,0 \% de pureza (Aldrich) se utilizó como agente de vulcanización.

\section{Métodos}

\section{Preparación de muestras}

Las formulaciones usadas se presentan en la Tabla 1. El hule SBR se acondicionó durante 5 minutos a 30 ${ }^{\circ} \mathrm{C}$ en un mezclador de cilindros Schwabenthan modelo Polymix 80T; después de este tiempo se le añadieron óxido de Zinc y ácido esteárico y se mezclaron con el hule durante 5 minutos: a continuación se añadió el agente acoplante respectivo, el cual también se mezcló durante 5 minutos; posteriormente se añadieron los aceleradores (Hexa y MBT) y después de 5 minutos de mezclado se añadió la fibra (sólo para la elaboración de los compósitos). Para homogeneizar la dispersión de ésta, también se mezcló por 5 minutos con los ingredientes y finalmente se añadió el azufre y se mezcló por 5 minutos.

Para vulcanizar los elastómeros, éstos fueron termoprensados en un equipo Carver modelo 3895. Las condiciones del termoprensado fueron: temperatura de prensado de $160{ }^{\circ} \mathrm{C}$, tiempo de prensado 7,5 minutos, presión de trabajo de $22000 \mathrm{kPa}$; los elastómeros vulcanizados se retiraron de la prensa cuando la temperatura disminuyó a $120^{\circ} \mathrm{C}$. Cuando éstos alcanzaron la temperatura ambiente, se utilizó un troquel para obtener las probetas adecuadas para su caracterización correspondiente. 
Tabla 1: Formulaciones de elastómeros y sus compósitos, con 7,5 phr de compatibilizante o agente de acoplamiento.

\begin{tabular}{|c|c|c|c|c|c|c|c|c|}
\hline Clave & $\begin{array}{c}\text { Compatibilizante } \\
\text { o Acoplante }\end{array}$ & $\begin{array}{c}\text { Fibra, } \\
\text { phr }\end{array}$ & $\begin{array}{c}\text { Azufre, } \\
\text { phr }\end{array}$ & $\begin{array}{l}\text { SBR } \\
\text { phr }\end{array}$ & $\begin{array}{l}\text { Óxido de } \\
\text { Zinc, phr }\end{array}$ & $\begin{array}{c}\text { Ácido } \\
\text { esteárico, } \mathrm{phr}\end{array}$ & $\begin{array}{c}\text { Hexa, } \\
\text { phr }\end{array}$ & $\begin{array}{c}\text { MBT, } \\
\text { phr }\end{array}$ \\
\hline IA & \multirow{6}{*}{ Amberyl A-30 } & \multirow{3}{*}{0} & 2,5 & 100 & 5 & 2 & 5 & 0,2 \\
\hline IB & & & 7,5 & 100 & 5 & 2 & 5 & 0,2 \\
\hline IC & & & 15 & 100 & 5 & 2 & 5 & 0,2 \\
\hline IA30 & & \multirow{3}{*}{30} & 2,5 & 100 & 5 & 2 & 5 & 0,2 \\
\hline IB30 & & & 7,5 & 100 & 5 & 2 & 5 & 0,2 \\
\hline IC30 & & & 15 & 100 & 5 & 2 & 5 & 0,2 \\
\hline IIA & \multirow{6}{*}{ R-HMT } & \multirow{3}{*}{0} & 2,5 & 100 & 5 & 2 & 5 & 0,2 \\
\hline IIB & & & 7,5 & 100 & 5 & 2 & 5 & 0,2 \\
\hline IIC & & & 15 & 100 & 5 & 2 & 5 & 0,2 \\
\hline IIA30 & & \multirow{3}{*}{30} & 2,5 & 100 & 5 & 2 & 5 & 0,2 \\
\hline IIB30 & & & 7,5 & 100 & 5 & 2 & 5 & 0,2 \\
\hline IIC30 & & & 15 & 100 & 5 & 2 & 5 & 0,2 \\
\hline
\end{tabular}

\section{Pruebas mecánicas}

Las pruebas de tracción se realizaron en un equipo de pruebas universales United Calibration Corporation 5802 Engineer Drive Testing Machine (modelo SFM-100), de acuerdo a la Norma ASTM-D412-16 (2016) a $25^{\circ} \mathrm{C}$ (utilizando probetas tipo C), a una velocidad de cruceta de $200 \mathrm{~mm} / \mathrm{min}$.

Las pruebas mecano-dinámicas se realizaron utilizando un equipo de TA Instruments modelo Q800-0824, con mordaza de cantiléver doble. Se utilizaron probetas rectangulares con dimensiones de 59,7 mm, 12,76 $\mathrm{mm}$ y 3,19 $\mathrm{mm}$. Para el barrido de temperatura, se utilizó un rango de temperatura de $-75^{\circ} \mathrm{C}$ a $40{ }^{\circ} \mathrm{C}$ con una velocidad de calentamiento de $2{ }^{\circ} \mathrm{C} / \mathrm{min}$ y una frecuencia de $1 \mathrm{~Hz}$. Las pruebas mecano-dinámicas, también se llevaron a cabo a temperatura ambiente haciendo un barrido de frecuencia de $0,4 \mathrm{~Hz}$ a $40 \mathrm{~Hz}$ bajo el mismo esquema de configuración de equipo y dimensiones de probetas.

\section{Microscopía electrónica de barrido (SEM)}

Para la caracterización morfológica, en los materiales procesados sin y con fibra, así como con agente acoplante o compatibilizante se hizo fractura criogénica en nitrógeno líquido para evitar cambios o deformaciones morfológicas en las muestras; posteriormente se recubrieron con oro en un pulverizador con vacío para observarlos (transversalmente) en un microscopio marca JEOL (modelo JCM-6000PLUS).

\section{RESULTADOS Y DISCUSIÓN}

En los resultados se presentan las pruebas de tracción, pruebas de análisis mecanodinámico (barridos de temperatura y frecuencia), en conjunto con la morfología (microscopía electrónica de barrido). Para comparar los dos sistemas; R-HMT y A30 como compatibilizante y agente acoplante.

Con respecto a propiedades mecánicas, en la Figura 2 se muestran las curvas de esfuerzo-deformación para los elastómeros sin y con fibra de agave. En la Figura 2a se presentan los elastómeros vulcanizados sin fibra; allí se pueden observar tres zonas en cada curva; en la primera, la pendiente inicial representa el módulo de Young, la cual se incrementa con el contenido de azufre; la segunda zona es una meseta que disminuye en longitud con el contenido de azufre y está relacionada con un desenrollamiento y estiramiento de las cadenas del elastómero y en la tercer zona aparece otra pendiente positiva, la cual vuelve a incrementarse con el con- 
tenido de azufre. Se puede también observar que los elastómeros en los que se utilizó el agente A-30 muestran en todos los casos valores mayores de módulo de Young, resistencia a la tracción y deformación, que en los que se utilizó el sistema R-HMT. En la Tabla 2 se muestran los valores del módulo de Young y el porcentaje de deformación, donde se puede observar que se tiene un incremento en el módulo de Young de 74 \%, 23 \% y 27 $\%$ para los elastómeros preparados con A-30, conteniendo $2,5 \mathrm{phr}, 7,5 \mathrm{phr}$ y $15 \mathrm{phr}$ de azufre respectivamente, con respecto a sus equivalentes preparados con resorcinol. Por otro lado, el incremento en la deformación de los elastómeros preparados con A-30 comparado con los elastómeros conteniendo resorcinol corresponde a $43 \%$, $260 \%$, y $13 \%$ para los contenidos en azufre de $2,5 \mathrm{phr}, 7,5 \mathrm{phr}$ y $15 \mathrm{phr}$ respectivamente. Las diferencias entre resorcinol y el agente A-30 que conducen a mejores resultados comparativos para los materiales elastoméricos con este último, residen en que por un lado la molécula de Amberyl tiene mucho mayor peso molecular, lo cual le da mayor estabilidad y capacidad de interacción/molécula) y por otro es más larga, con lo cual ofrece mayor capacidad de cobertura e interacción con la matriz; esto es, se logra una mejor integración con los ingredientes del material cuando se le aplica un esfuerzo. Ello se fortalece adicionalmente, porque el Amberyl contiene más grupos metilo y anillos en su estructura, que presentan afinidad con las estructuras de butadieno y estireno del elastómero SBR. Todo ello influye para tener un incremento en el módulo de Young y en la capacidad de deformación (comparando con el papel que juega el par R-HMT).

Por otro lado, con la adición de $30 \mathrm{phr}$ de fibra de agave, se modifica la forma de las curvas de esfuerzo-deformación comparadas con las muestras sin agente reforzante. Aumentan los módulos y disminuye considerablemente la capacidad de deformación. Sin embargo, la superioridad en desempeño del agente A-30 sobre el resorcinol también se refleja en los valores de módulo y capacidad de deformación (aunque ésta disminuye en ambos tipos de compósitos) de los materiales, tal como se puede ver en la Figura $2 \mathrm{~b}$ y en la Tabla 2. La orientación de las cadenas de elastómero, indicada por el aumento en la pendiente al aumentar la deformación (zona tres en las curvas de Figura 2a) ya no se muestra. Se presenta un punto de inflexión con una disminución del esfuerzo al seguir deformándose, que es referido como esfuerzo de cedencia, que es característico de los hules reforzados con fibras (Wang et al. 2011); dicho punto indica la terminación del comportamiento elástico del material y el inicio de deformación permanente, iniciándose una separación en la interfaz entre la fibra de agave y la matriz de hule. Puede observarse que el punto de cedencia está influido por el tipo de agente de acoplamiento y que el contenido de azufre influye en la deformación última. Para el caso de los sistemas elastoméricos con carga de negro de humo, el fenómeno de cedencia a baja deformación, se ha reportado que está relacionado con la descomposición de los agregados (Suzuki et al. 2002).

Después del límite elástico en la Figura 2b, el efecto de refuerzo de la fibra de agave básicamente desaparece y la resistencia y deformación remanente se deberá fundamentalmente al material elastomérico (Wang et al. 2011).

Por otra parte, se obtienen mayores valores del módulo de Young y de la deformación de los compósitos en los que se utilizó A-30 comparando con los materiales que contienen R-HMT, como se puede observar en la Tabla 2. Entre ellos, el módulo que resalta es el de la formulación con menor cantidad de azufre; el incremento es de un $38 \%$ cuando se usa el A-30 comparado con la formulación con R-HMT. Al haber un alto contenido de fibra e incrementar el grado de reticulación con el contenido de azufre, se observa que el módulo de Young se incrementa y la deformación disminuye. Esto es debido a que las cadenas poliméricas pueden moverse menos. Suzuki et al. (2002), han sugerido que las moléculas poliméricas pierden su esencia como elastómero y se comportan como un relleno flexible en términos de propiedades de tracción-deformación al estar entre fibras. No obstante, tanto la mayor longitud molecular como la cantidad de grupos funcionales (oxihidrilo y éster) presentes en el A-30, permiten establecer mayor cantidad de puentes de hidrógeno con los grupos $\mathrm{OH}$ de la fibra de agave. Lo anterior permite explicar el por qué los materiales en los que se utilizó el Amberyl, presentan mayor módulo y mayor deformación que los equivalentes preparados con R-HMT (Tabla 2).

El efecto del peso molecular del agente acoplante en las propiedades mecánicas, también se ha reportado utilizando polietilenos maleinizados (MAPE) como agentes de acoplamiento de fibra de pulpa de madera. La resistencia a la tracción se incrementó al aumentar el peso molecular del MAPE (Lu et al. 2005). Con respecto a la disminución de la capacidad de deformación al estar presente la fibra, se ha determinado que al aumentar el contenido de fibra celulósica puede disminuir la deformación en SBR hasta en un $75 \%$, cuando se utiliza un $15 \%$ de fibra (Raza et al. 2016), o en hule de llanta con sisal, disminuir la deformación a un $45 \%$ con 20 $\%$ de fibra (Martins y Mattoso 2004). De manera similar, con el mismo tipo de hule y fibra corta de desecho de llanta reciclada, la deformación bajó a $50 \%$ con $15 \%$ de fibra (Zhang et al. 2007). Resultados similares usando hule nitrílico con fibra de polvo de piña fueron reportados hace pocos años, habiéndose determinado que de $400 \%$ de deformación sin fibra, con $20 \%$ y el $30 \%$ de fibra, su capacidad de deformación disminuyó a aproximadamente $35 \%$ con el primero y $25 \%$ con la mayor cantidad de fibra (Wisittanawat et al. 2014). 

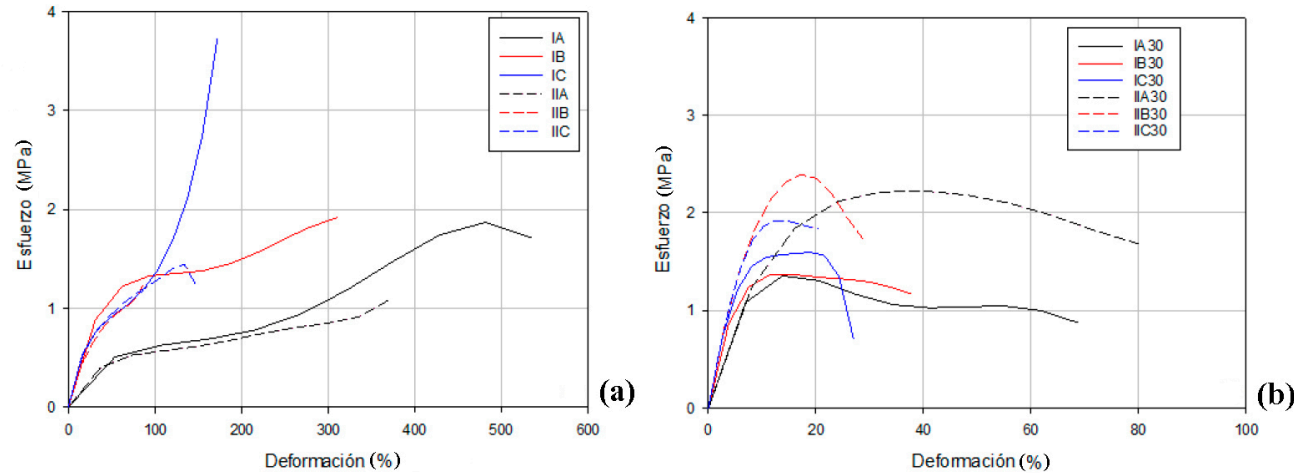

Figura 2: Comportamiento de esfuerzo-deformación de los materiales elastoméricos y compósitos de SBR a $25^{\circ} \mathrm{C}$, con diferentes contenidos de azufre: (a) $0 \mathrm{phr}$ de fibra de agave, (b) $30 \mathrm{phr}$ de fibra de agave.

Nota: En Tabla 1 código de materiales.

Tabla 2: Propiedades mecánicas de los diferentes materiales elastoméricos de SBR y sus compósitos.

\begin{tabular}{|c|c|c|c|c|c|c|c|}
\hline \multicolumn{2}{|c|}{ Contenido de material } & \multicolumn{2}{|c|}{ Módulo de Young, MPa } & \multicolumn{2}{c|}{$\%$ de deformación } & \multicolumn{2}{c|}{$\begin{array}{c}\text { de incremento de A30 } \\
\text { sobre R-HMT }\end{array}$} \\
\hline $\begin{array}{c}\text { Fibra, } \\
\text { phr }\end{array}$ & $\begin{array}{c}\text { Azufre, } \\
\text { phr }\end{array}$ & R-HMT & A-30 & R-HMT & A-30 & $\begin{array}{c}\text { Módulo } \\
\text { de } \\
\text { Young }\end{array}$ & $\begin{array}{c}\% \text { de } \\
\text { deformación }\end{array}$ \\
\hline \multirow{3}{*}{0} & 2,5 & $2,4 \pm 0,05$ & $4,1 \pm 0,51$ & $373,5 \pm 19,7$ & $535,7 \pm 115$ & 74,15 & 43,43 \\
\cline { 2 - 8 } & 7,5 & $3,6 \pm 0,13$ & $4,5 \pm 0,30$ & $86,2 \pm 14,8$ & $310,5 \pm 18,2$ & 23,08 & 260,21 \\
\hline \multirow{3}{*}{30} & 15 & $4,7 \pm 0,18$ & $5,9 \pm 0,44$ & $149,1 \pm 14,7$ & $167,9 \pm 14,4$ & 26,82 & 12,61 \\
\cline { 2 - 8 } & 2,5 & $17,2 \pm 1,31$ & $23,8 \pm 2,1$ & $77,7 \pm 14,4$ & $68,8 \pm 20,1$ & 38,02 & $-11,45$ \\
\cline { 2 - 8 } & 7,5 & $24,9 \pm 1,79$ & $26,7 \pm 2,9$ & $29,8 \pm 2,4$ & $38,1 \pm 7,6$ & 7,48 & 27,85 \\
\hline
\end{tabular}

Con respecto a las propiedades mecano-dinámicas, en la Figura 3 se muestra el módulo de almacenamien-to (E') como función de la temperatura, para los materiales preparados sin fibra (Figura 3a) y con fibra (Figura $3 \mathrm{~b}$ ). Allí se puede observar, que para E' a temperaturas menores a $-40{ }^{\circ} \mathrm{C}$, los materiales sin fibra de agave muestran valores entre $5000 \mathrm{MPa}$ y $10000 \mathrm{MPa}$ mientras que al contener fibra los valores están entre $2000 \mathrm{MPa}$ y $4000 \mathrm{MPa}$. Ello indica que cuando los materiales están por debajo de la temperatura de transición vítrea, los materiales "homogéneos" (sin fibra), tienen mayor capacidad de almacenar energía que los heterogéneos (con fibra). En la meseta a baja temperatura, los materiales son rígidos con comportamiento tipo vítreo (rango térmico sin utilidad como elastómeros); en ausencia de fibra las cadenas de hule pueden compactarse más que la del agente A-30, y por ello presentan mayor E' que los materiales equivalentes con dicho agente. El mayor contenido de azufre también influye en un mayor valor de E'. Sin embargo, con la presencia de fibra, el efecto de compactación a baja temperatura se disminuye por la interferencia física de dichas fibras (material heterogé-neo), obteniéndose un menor valor de E' en tales materiales. A temperaturas entre $-40{ }^{\circ} \mathrm{C}$ y $-20{ }^{\circ} \mathrm{C}$ se tiene una fuerte caída de $\mathrm{E}$ ' que representa la temperatura de transición vítrea $\left(\mathrm{T}_{\mathrm{g}}\right)$ de la matriz, la cual es mayor para los materiales con mayor cantidad de reticulante; la mayor capacidad de interacción del agente A-30 se manifiesta promoviendo que la caída de E' se presente a mayor temperatura en los materiales con A-30 que con R-HMT (tanto sin como con fibra). Siguiendo luego la trayectoria de las curvas, se presentan las mesetas ahuladas co-rrespondientes (zona térmica de aplicación como compósitos a base de elastómero) y se puede observar para los materiales sin fibra de agave a temperaturas entre $20^{\circ} \mathrm{C}$ y $30^{\circ} \mathrm{C}$, que el E' está en el rango de $10 \mathrm{MPa}$ a los $50 \mathrm{MPa}$ mientras que para los materiales reforzados con fibra se encuentra entre los $40 \mathrm{MPa}$ y $100 \mathrm{MPa}$.

En general se puede apreciar para las diferentes formulaciones, que los hules en los que se utilizó la resina pinífera esterificada como agente compatibilizante presentan un mayor $\mathrm{E}$ ' a temperaturas mayores a $0{ }^{\circ} \mathrm{C}$, comparados con los materiales equivalentes en los que se utilizó R-HMT. Además, para los materiales sin reforzar, se puede observar en la meseta de comportamiento ahulado, que al aumentar la temperatura, las líneas se van juntando a partir de $0{ }^{\circ} \mathrm{C}$. Algo similar ocurre en los materiales con fibra. En tales circunstancias, el orden de acomodo de los valores de E', ya no se mantiene con respecto a la cantidad de reticulante en ambas series. Un 
hecho a resaltar utilizando el agente A-30 con fibra, es que el mayor valor de E' corresponde al material con la menor cantidad de azufre. Ello puede indicar que al aumentar la temperatura y con ello la movilidad molecular, si hay buena interacción entre los componentes, la capacidad de alineación es mayor para las cadenas con menor cantidad de reticulante, provocando un incremento en el módulo que no se presenta marcadamente por alineamiento si aumenta la reticulación. Ante tal situación, se puede tener una mejor respuesta para bajos valores de módulo, haciendo la medición a temperatura constante con un barrido de frecuencia para validar comportamientos.

Dicha prueba se muestra en la Figura 4, donde puede observarse que las curvas a baja frecuencia están claramente separadas, y que a frecuencia de $1 \mathrm{~Hz}$ (que es la que se usa con el barrido de temperatura) puede notarse para los materiales sin fibra y con fibra, que con R-HMT sí mantienen el orden de que a mayor reticulación, se presenta mayor E'; de igual forma, para el uso del agente A-30 los materiales con mayor módulo son los que tienen mayor cantidad de reticulante, mientras que con $2,5 \mathrm{phr}$ y 7,5 phr de azufre, los valores son prác-ticamente iguales. Nuevamente para materiales equivalentes, los materiales con el agente A-30 superan a los preparados con R-HMT (en un $46 \%, 28 \%$ o $19 \%$ para contenidos de 2,5 phr, 7,5 phr o $15 \mathrm{phr}$ de azufre res-pectivamente). Lo que también puede verse, es que al aumentar la frecuencia, los materiales presentan un in-cremento en E'. Sin embargo, la pendiente positiva es mayor para las líneas con menor cantidad de reticulante. Ello significa que el incremento en E' para los materiales, no sólo se debe a que al aumentar la frecuencia dis-minuye el tiempo de relajación entre ciclo y ciclo de aplicación de esfuerzo (esto es, la relajación de esfuerzos hace que el módulo se mantenga en bajo valor), sino que también indica que con menor cantidad de reticulante, las cadenas pueden alinearse más, promoviendo un mayor incremento en el módulo. Ello puede notarse para los compósitos ahulados tanto sin fibra como con fibra, aunque el valor de la pendiente positiva es menor con la presencia de fibra porque la capacidad de alineación molecular es menor.
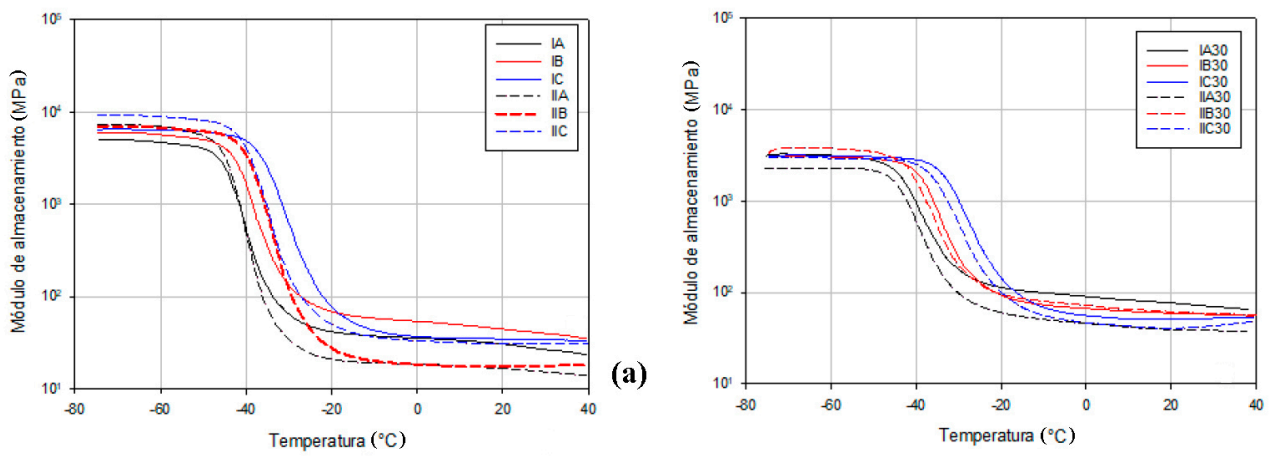

Figura 3: Módulo de almacenamiento de materiales elastoméricos y compósitos de SBR como función de la temperatura, para diferentes contenidos de azufre: (a) $0 \mathrm{phr}$ de fibra de agave, (b) $30 \mathrm{phr}$ de fibra de agave Nota: En Tabla 1 código de materiales.
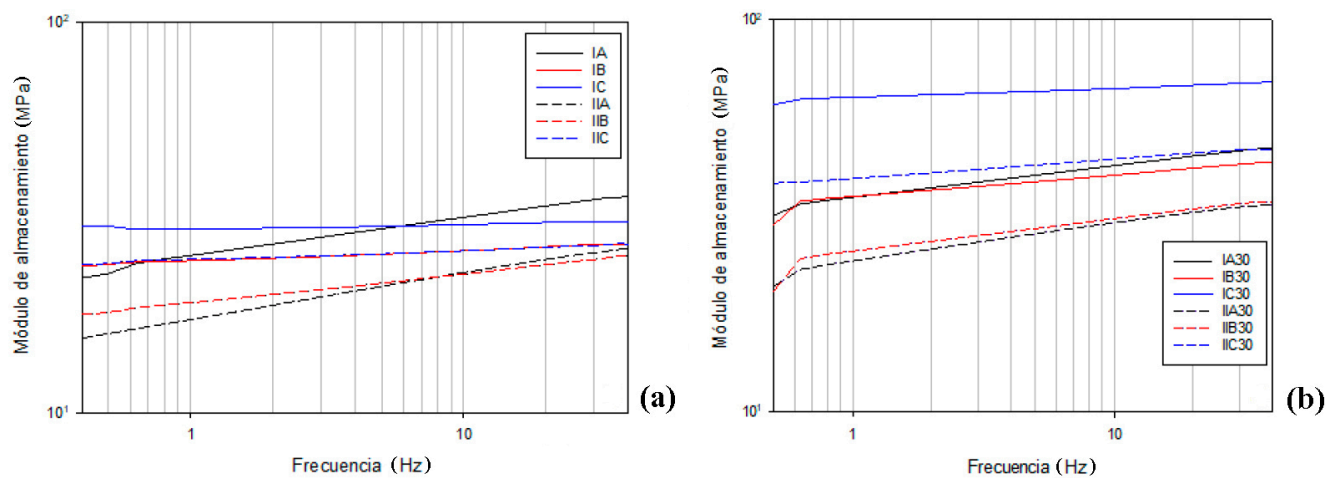

Figura 4: Módulo de almacenamiento a $30^{\circ} \mathrm{C}$ de materiales elastoméricos y compósitos de SBR como función de la frecuencia, para diferentes contenidos de azufre: (a) $0 \mathrm{phr}$ de fibra de agave, (b) $30 \mathrm{phr}$ de fibra de agave.

Nota: En Tabla 1 código de materiales. 
En la Figura 5, se muestra el módulo de pérdida (E”) como función de la temperatura, mientras que en la Tabla 3 se muestran las $\mathrm{T} s$ representadas por el valor máximo de E" del pico correspondiente. En la Figura 5a se puede observar que la $\mathrm{T}_{\mathrm{g}}$ se recorre a la derecha al incrementar el contenido de azufre, debido al mayor entrecruzamiento de las moléculas del hule; lo cual se presenta también con presencia de fibra (Figura 5b). Los valores de $\mathrm{T}_{\mathrm{g}}$ para los materiales equivalentes, son ligeramente mayores para los que contienen el agente A-30, lo cual concuerda con los valores de la caída correspondiente de E' debido a su mayor capacidad de interacción tanto sin como con fibra.

Tabla 3: Módulo de almacenamiento a $30^{\circ} \mathrm{C}$ obtenidos de la Figura 4 y $\mathrm{T}_{\mathrm{g}}$ de los materiales de $\mathrm{SBR}$ sin fibra y de los compósitos.

\begin{tabular}{|c|c|c|c|c|c|c|}
\hline \multirow{2}{*}{\multicolumn{2}{|c|}{$\begin{array}{l}\text { Contenido de } \\
\text { material }\end{array}$}} & \multirow{2}{*}{\multicolumn{2}{|c|}{$\mathrm{E}^{\prime} \mathrm{MPa}$}} & & \multirow{2}{*}{\multicolumn{2}{|c|}{$\frac{\mathrm{T}_{\mathrm{g}},{ }^{\circ} \mathrm{C}}{\mathrm{E}{ }^{\circ}}$}} \\
\hline & & & & \multirow{2}{*}{$\begin{array}{c}\% \text { de } \\
\text { incremento }\end{array}$} & & \\
\hline $\begin{array}{c}\text { Fibra, } \\
\text { phr }\end{array}$ & $\begin{array}{c}\text { Azufre, } \\
\text { phr }\end{array}$ & R-HMT & A-30 & & R-HMT & A-30 \\
\hline \multirow{3}{*}{0} & 2,5 & 17,30 & 25,22 & 45,74 & $-44,2$ & $-44,2$ \\
\hline & 7,5 & 19,11 & 44,46 & 28,01 & $-38,61$ & $-41,15$ \\
\hline & 15 & 24,66 & 29,47 & 19,54 & $-39,37$ & $-35,57$ \\
\hline \multirow{3}{*}{30} & 2,5 & 24,19 & 36,53 & 51,03 & $-42,42$ & $-42,7$ \\
\hline & 7,5 & 25,62 & 35,34 & 37,96 & $-40,64$ & $-37,35$ \\
\hline & 15 & 39,30 & 63,34 & 61,17 & $-34,57$ & $-31,77$ \\
\hline
\end{tabular}

*Valor tomado del pico de E” de la Figura 5.

Por otra parte, con la adición de la fibra natural puede verse que disminuye el rango de valores pico del módulo correspondiente y con ello la capacidad de disipación energética del material. El área bajo la curva entre $-60{ }^{\circ} \mathrm{C}$ y $30{ }^{\circ} \mathrm{C}$ baja en un $46,5 \%$ en promedio, de sin fibra a con fibra. Dicha disminución se debe primordialmente a que el caucho sin fibra es muy flexible (con alta capacidad de disipación energética), pero con la presencia de fibra generando el compósito, se introduce algo de rigidez y cristalinidad por el elevado por-centaje de celulosa en la fibra de agave (Pérez-Fonseca 2014); adicionalmente, la fibra dificulta la movilidad de las moléculas de hule disminuyendo la capacidad de amortiguación del material.

En la Tabla 3 se muestran las temperaturas de transición vítrea $\left(\mathrm{T}_{\mathrm{g}}\right)$ de los diferentes materiales. Se puede observar que la $\mathrm{T}_{\mathrm{g}}$ se incrementa con el contenido de azufre para todos los casos. Además, se puede ver que los elastómeros con agente A-30 presentan la misma $T_{g}$ o mayor que en los que utilizó el resorcinol y se muestra que la $\mathrm{T}_{\mathrm{g}}$ es mayor para los hules reforzados con fibra de agave que sin ella. Tanto la adición de azufre como de fíbra logran impedir el movimiento segmental de las moléculas del hule; por otra parte, la mayor capacidad de interacción del agente A-30 queda manifiesta.
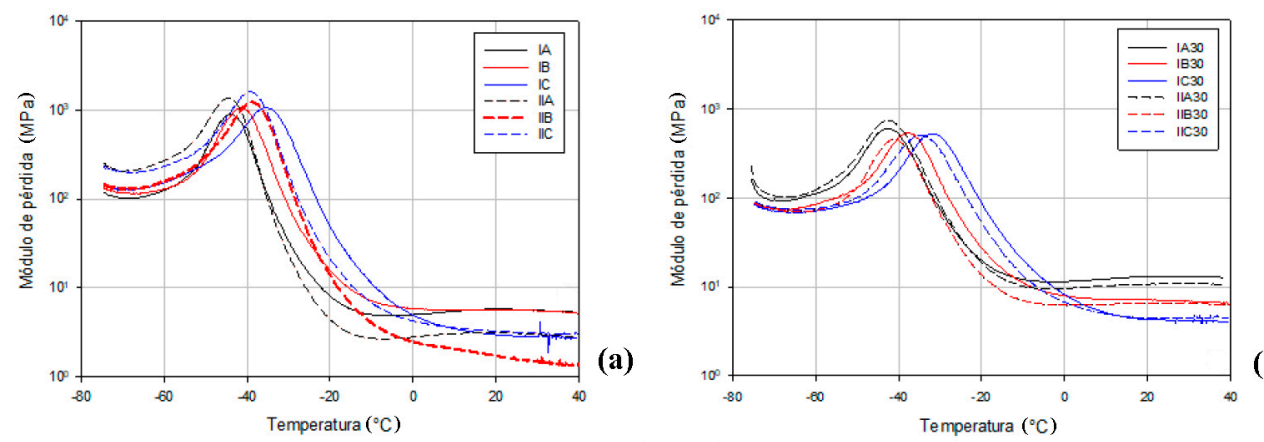

(b)

Figura 5: Módulo de pérdida de materiales elastoméricos y compósitos de SBR como función de la tempera-tura, con diferentes contenidos de azufre: (a) $0 \mathrm{phr}$ de fibra de agave, (b) $30 \mathrm{phr}$ de fibra de agave. Nota: En Tabla 1 código de materiales. 
En la Figura 6 se muestra la variación del módulo de pérdida con respecto a la frecuencia. Allí se puede observar, que para un incremento de ésta, tanto sin fibra como con ella, aumenta el E" del material correspondiente. Se puede notar para los hules sin fibra (Figura 6a), que los materiales con menor cantidad de reticulante tienen mayor módulo, mostrando mayor capacidad de disipación energética. Por otra parte, en los compósitos (Figura 6b) se puede apreciar al aumentar la frecuencia, que la pendiente positiva es menor que para los materiales equivalentes sin fibra, indicando que las fibras largas disminuyen la movilidad de las moléculas del hule.
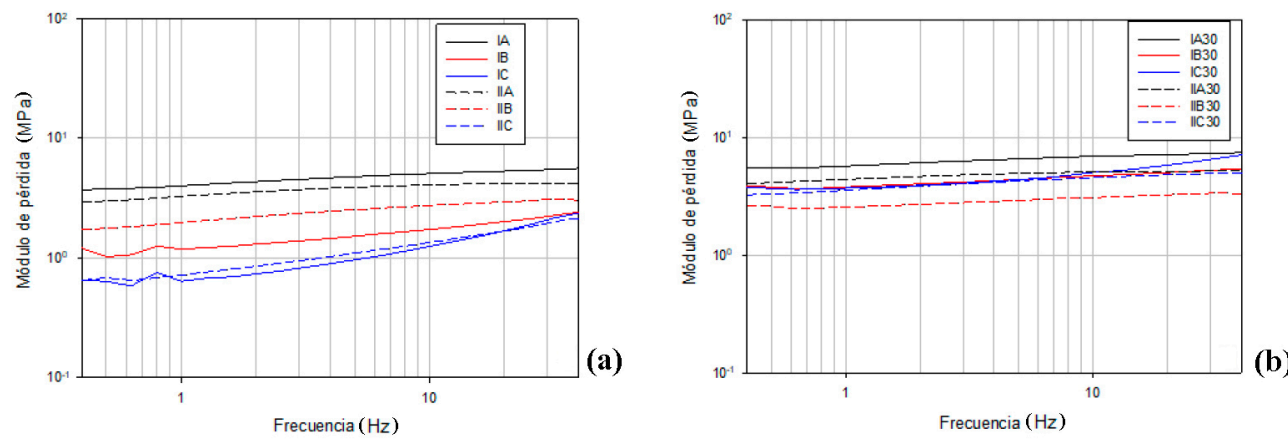

Figura 6: Módulo de pérdida a $30^{\circ} \mathrm{C}$ como función de la frecuencia para materiales elastoméricos y compósitos de SBR para diferentes contenidos de azufre: (a) $0 \mathrm{phr}$ de fibra de agave, (b) $30 \mathrm{phr}$ de fibra de agave. Nota: En Tabla 1 código de materiales.

En la Figura 7 se muestran las imágenes de los materiales tomadas con un microscopio electrónico de barrido a 100X. Las imágenes Figura 7a y Figura 7b representan la matriz de SBR con el agente A-30 conteniendo 2,5 phr y $15 \mathrm{phr}$ de azufre respectivamente. En las imágenes Figura 7c y Figura 7d, se muestran los materiales equivalentes utilizando resorcinol como acoplante. En dichas figuras, se puede observar para ambos agentes de acoplamiento que la compacidad (referida a tener menos huecos en la masa) y rugosidad se incrementan con el contenido de azufre, siendo más significativas las diferencias con el agente A-30 que con R-HMT. Ello puede ser indicativo de una mayor interacción entre el agente acoplante y la matriz, que luego tiene un efecto en las propiedades mecánicas.
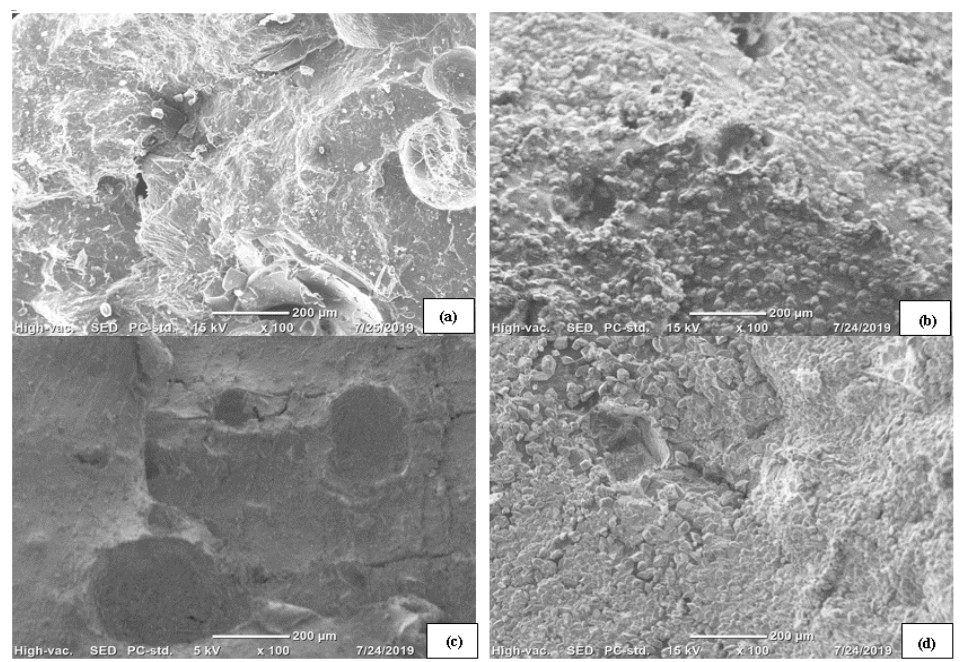

Figura 7: Micrografías de elastómeros de SBR sin fibra. Agente de acoplamiento: A-30 para (a) y (b); R-HMT para (c) y (d). Contenido de azufre en phr: 2,5 para (a) y (c), 15 para (b) y (d).

Las imágenes de los materiales con fibra de agave fueron tomadas utilizando muestras fracturadas criogénicamente (para no afectar las muestras al romperlas), para poder observar la diferencia en poder acoplante entre el agente A-30 y el R-HMT. Con respecto a la Figura 8, en las imágenes Figura 8a, Figura 8b, Figura 8c, Figura $8 \mathrm{~d}$ se puede notar lo siguiente: con bajo contenido de azufre $(2,5 \mathrm{phr})$; la superficie del SBR sigue siendo menos rugosa con ambos agentes compatibilizantes (Figura 8a) con agente A-30 y (Figura 8c) con R-HMT, que con contenidos elevados de azufre (Figura 8b) con agente A-30 y (Figura 8d) con R-HMT. Igualmente 
puede notarse que sí existe capacidad de acoplamiento con ambos tipos de agentes, ya que con bajo contenido de azufre la rotura de fibras es de tipo longitudinal, lo cual indica que éstas presentan resistencia a la separación de la matriz (Figura 8a) y (Figura 8c), a la vez que se muestra cierta capacidad de deformación, mientras que la fractura de las fibras es prácticamente perpendicular al tener un elevado contenido de azufre (Figura 8b) y (Figura 8d). Además, es importante resaltar que tanto con bajo como con alto contenido de azufre, en la interfase fibra-matriz puede notarse una mayor separación en las muestras con R-HMT que en sus equivalentes con fibra de agave. Tal diferencia es relevante porque permite observar la mayor capacidad como acoplante del agente A-30 al generar una mayor compactación logrando disminuir la separación entre fibra y matriz, lo cual redunda en una mayor resistencia mecánica (mostrando mayor dificultad de separación al aumentar la deformación). Las fotografías confirman entonces, la mayor capacidad de interacción que este agente ofrece como compatibilizante y acoplante en los respectivos materiales.
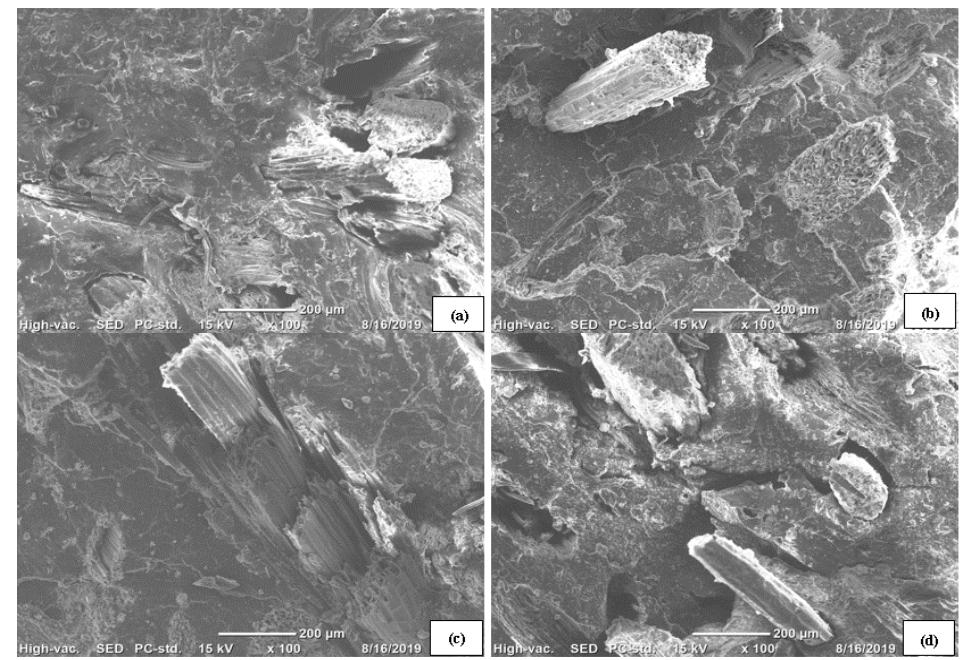

Figura 8: Micrografías de compósitos de SBR con $30 \mathrm{phr}$ de fibra de agave. Agente de acoplamiento: A-30 para (a) y (b); R-HMT para (c) y (d). Contenido de azufre en phr: 2,5 para (a) y (c), 15 para (b) y (d).

\section{CONCLUSIONES}

Los resultados obtenidos en propiedades mecano-estáticas y mecano-dinámicas muestran que los valores medidos son mejores al utilizar una resina pinífera modificada en lugar de R-HMT, tanto en un compósito con fibra de agave y hule de SBR, como en la formulación sin fibra. Ello demuestra que sí es factible y conveniente elaborar elastómeros utilizando la resina pinífera esterificada ya sea sin reforzante o como compósitos reforzados con fibra de agave (lo cual también puede ser posible con otras fibras celulósicas). Así entonces, el uso de ingredientes verdes para sustituir elementos tóxicos y/o contaminantes (aquí el agente A-30 en lugar de R-HMT) permite hacer formulaciones y compósitos que sean más amigables con el medio ambiente, manteniendo buenas propiedades mecánicas o incluso incrementarlas.

Como aportación para el mejoramiento de este tipo de materiales, se demostró que con los elastómeros puros preparados usando la resina de pino maleinizada, los módulos de Young y de almacenamiento, además de la capacidad de deformación y de disipación energética son considerablemente mayores que los preparados con R-HMT. Dicha superioridad se sostuvo al incluir la fibra de agave, aunque la diferencia en propiedades disminuyó con la presencia de la fibra que fue usada en ambos tipos de compósitos.

Para los materiales con la mayor capacidad elastomérica (usando la menor cantidad de reticulante), tanto sin fibra como con fibra de agave, se pueden preparar formulaciones para aplicaciones que requieran de elevada tenacidad.

La superioridad como acoplante del agente A-30 con respecto al R-HMT, se pudo reflejar con claridad morfológicamente por presentar mayor compacidad en la matriz y mayor cercanía entre fibra y matriz en muestras fracturadas. 


\section{REFERENCIAS}

Abd-El-Messieh, S.L.; Abd-El-Nour, K.N. 2003. Effect of curing time and sulfur content on the dielectric relaxation of styrene butadiene rubber. J Appl Polym Sci 88(7): 1613-1621. https://doi.org/10.1002/app.11686

ASTM. 2016. ASTM-D412-16: Standard Test Methods for Vulcanized Rubber and Thermoplastic Elastomers-Tension. West Conshohocken, PA, USA. http://www.astm.org/cgi-bin/resolver.cgi?D412-16

Ciesielski, A. 1999. An introduction to rubber technology. Rapra Technology Limited: Shrewsbury, Shropshire, Great Britain.

Consejo Regulador del Tequila. 2019. Reporte 2019. https://www.crt.org.mx/EstadisticasCRTweb/

Debasish, D.; Debapriya, D.; Adhikari, B. 2006. Curing characteristics and mechanical properties of alkali-treated grass-fiber-filled natural rubber composites and effects of bonding agent. J Appl Polym Sci 101(5): 3151-3160. https://doi.org/10.1002/app.23305

Dressler, H. 1994. The Use of Resorcinol in Rubber Compositions. In Resorcinol. Topics in Applied Chemistry. Springer: Boston, MA, USA. https://doi.org/10.1007/978-1-4899-0999-2_4

El-Wakil, A.A. 2011. Enhancement of adhesion between EPDM and polyester fabric by using natural rubber modified by maleic anhydride. Int J Polym Sci 2011: 1-5. https://doi.org/10.1155/2011/591948

Flickinger, C.W. 1976. The benzenediols: catechol, resorcinol and hydroquinone-a review of the industrial toxicology and current industrial exposure limits. Am Ind Hyg Assoc J 37(10): 596-606. https://doi.org/10.1080/0002889768507526

Gallo-Corredor, J.A.; Sarria-Villa, R. 2013. Obtención de colofonia y trementina a partir de resina de pino de la especie patula y posterior evaluación de los parámetros de calidad. J Ciencia Ing 5(1): 88-91. https://jci.uniautonoma.edu.co/2013/2013-13.pdf

Geethamma, V.G.; Joseph, R.; Thomas, S. 1995. Short coir fiber-reinforced natural rubber composites: Effects of fiber length, orientation, and alkali treatment. J Appl Polym Sci 55(4): 583-594. https://doi.org/10.1002/app.1995.070550405

Geethamma, V.G.; Kalaprasad, G.; Groeninckx, G.; Thomas, S. 2005. Dynamic mechanical behavior of short coir fiber reinforced natural rubber composites. Compos Part A Appl Sci Manuf 36(11): 1499-1506. https://doi.org/10.1016/j.compositesa.2005.03.004

Halim, S.F.; Lawandy, S.N.; Nour, M.A. 2013. Effect of in situ bonding system and surface modification of montmorillonite on the properties of butyl rubber/montmorillonite composites. Polym Compos 34(9): 15591565. https://doi.org/10.1002/pc. 22573

Inga-Lafebre, J.; Pulido-González, H.; González-Núñez, R.; Hernández-Hernández, M.E.; Rabelero-Velasco, M.; Aranda-García, F.J.; Jasso-Gastinel, C.F. 2019. The Multirole of Modified Natural Gums for Multicomponent Polymers: As Coupling Agents for Polymers Reinforced With Cellulosic Fibers or Compatibilizers for Biodegradable Polymer Blends. Quim Nova 42(3): 296-304. https://doi.org/10.21577/01004042.20170333

Jacob, M.; Francis, B.; Thomas, S.; Varughese, K.T. 2006. Dynamical mechanical analysis of sisal/oil palm hybrid fiber-reinforced natural rubber composites. Polym Compos 27(6): 671-680. https://doi.org/10.1002/pc. 20250

Jacob, M.; Thomas, S.; Varughese, K.T. 2004. Mechanical properties of sisal/oil palm hybrid fiber reinforced natural rubber composites. Compos Sci Technol 64(7-8): 955-965. https://doi.org/10.1016/S02663538(03)00261-6

Jasso-Gastinel, C.F.; Lopez-Carrillo, P.; Mendizabal-Mijares, E. 1992. Mechanical and rheological properties of poly(butadiene-acrylonitrile) rubber compounds reinforced with cellulosic material. Polym Eng Sci 32(6): 443-447. https://doi.org/10.1002/pen.760320610 
Jiang, W.; Gu, J. 2020. Nanocrystalline cellulose isolated from different renewable sources to fabricate natural rubber composites with outstanding mechanical properties. Cellulose 27: 5801-5813. https://doi.org/10.1007/s10570-020-03209-3

Lawandy, S.N.; Moustafa, H.; Zahran, M.A.; Rabee, M. 2018. Effect of bio-alkyd resin oil content and viscosity on the adhesion of EPDM to polyester fabric. J Adhes Sci Technol 32(3): 302-316. https://doi.org/10 $.1080 / 01694243.2017 .1354668$

Lin, Y.; Liu, S.; Peng, J.; Liu, L. 2016. The filler - rubber interface and reinforcement in styrene butadiene rubber composites with graphene / silica hybrids : A quantitative correlation with the constrained region. Compos Part A Appl Sci Manuf 86: 19-30. https://doi.org/10.1016/j.compositesa.2016.03.029

Liu, X.; Zhao, S.; Zhang, X.; Li, X.; Bai, Y. 2014. Preparation, structure, and properties of solution-polymerized styrene-butadiene rubber with functionalized end-groups and its silica-filled composites. Polymer 55(8): 1964-1976. https://doi.org/10.1016/j.polymer.2014.02.067

López-Manchado, M.A.; Arroyo, M. 2002. Short fibers as reinforcement of rubber compounds. Polym Compos 23(4): 666-673. https://doi.org/10.1002/pc.10466

Lu, J.Z.; Wu, Q.; Negulescu, I.I. 2005. Wood-fiber/high-density-polyethylene composites: Coupling agent performance. J Appl Polym Sci 96(1): 93-102. https://doi.org/10.1002/app.21410

Martins, M.A.; Mattoso, L.H.C. 2004. Short sisal fiber-reinforced tire rubber composites: Dynamical and mechanical properties. $J$ Appl Polym Sci 91(1): 670-677. https://doi.org/10.1002/app.13210

Meissner, N.; Rzymski, W.M. 2013. Use of short fibers as a filler in rubber compounds. Autex Res $J$ 13(2): 40-43. https://doi.org/10.2478/v10304-012-0025-5

Mohanty, A.K.; Misra, M.; Drzal, L.T. 2001. Surface modifications of natural fibers and performance of the resulting biocomposites: An overview. Compos Interfaces 8(5): 313-343. https://doi.org/10.1163/156855401753255422

Pantamanatsopa, P.; Ariyawiriyanan, W.; Meekeaw, T.; Suthamyong, R.; Arrub, K.; Hamada, H. 2014. Effect of modified jute fiber on mechanical properties of Green rubber composite. Energy Procedia 56: 641-647. https://doi.org/10.1016/j.egypro.2014.07.203

Parambath-Kanoth, B.; Thomas, T.; Joseph, J.M.; Narayanankutty, S.K. 2019. Restructuring of coir to microfibers for enhanced reinforcement in natural rubber. Polym Compos 40(1): 414-423. https://doi.org/10.1002/pc.24667

Pérez-Fonseca, A.A.; Robledo-Ortíz, J.R.; Ramirez-Arreola, D.E.; Ortega-Gudiño, P.; Rodrigue, D.; González-Núñez, R. 2014. Effect of hybridization on the physical and mechanical properties of high density polyethylene-(pine/agave) composites. Mater Des 64: 35-43. https://doi.org/10.1016/j.matdes.2014.07.025

Poletto, M. 2017. Polypropylene-based wood-plastic composites: Effect of using a coupling agent derived from a renewable resource. Maderas-Cienc Tecnol 19(3): 265-272. https://doi.org/10.4067/S0718$221 \times 2017005000022$

Pulido-González, H.; Hernandez, E.; Rabelero Velasco, M.; Sanjuan Raygoza, R.J.; Jasso-Gastinel, C.F. 2014. Valoración mecanotérmica de una resina biodegradable como agente de acoplamiento de materiales compuestos celulósicos/polímero hidrofóbico. Maderas-Cienc Tecnol 16(4): 463-486. https://doi.org/10.4067/ S0718-221X2014005000038

Quiroz-Carranza, J.A.; Magaña-Alejandro, M.A. 2015. Natural resins of Mexican plant species: Current and potential end-uses. Madera y Bosques 21(3): 171-183. https://www.redalyc.org/articulo. oa? id $=61743002013$

Raza, M.A.; Ashraf, M.A.; Westwood, A.V.K.; Jamil, T.; Ahmad, R.; Inam, A.; Deen, K.M. 2016. Maleated high oleic sunflower oil-treated cellulose fiber-based styrene butadiene rubber composites. Polym Compos 37(4): 1113-1121. https://doi.org/10.1002/pc.23273 
Seo, G. 1997. Stabilizing the adhesion interphase between rubber compounds and brass film by the addition of resorcinol formaldehyde resin to the rubber. J Adhes Sci Technol 11(11): 1433-1445. https://doi.org/10.1163/156856197X00228

Shahril, D.A.; Azammi, A.M.N.; Zulfadli, S.M. 2017. Reinforcement of kenaf fiber in natural rubber composite for automotive engine rubber mounting. Int J Appl Eng Res 12(24): 14490-14494. http://www.ripublication.com/ijaer17/ijaerv12n24_70.pdf

Soltani, S.; Naderi, G.; Mohseniyan, S. 2014. Mechanical, morphological and rheological properties of short nylon fiber reinforced acrylonitrile-butadiene rubber composites. Fibers Polym 15(11): 2360-2369. https://doi.org/10.1007/s12221-014-2360-8

Sousa, D.; Biscaia, S.; Viana, T.; Gaspar, M.; Mahendra, V.; Mohan, S.D.; Mateus, A.; Mitchell, G.R. 2019. Rosin Based Composites for Additive Manufacturing. Appl Mech Mater 890: 70-76. https://doi.org/10.4028/www.scientific.net/amm.890.70

Suzuki, N.; Yatsuyanagi, F.; Ito, M.; Kaidou, H. 2002. Effects of surface chemistry of silica particles on secondary structure and tensile properties of silica-filled rubber systems. J Appl Polym Sci 86(7): 1622-1629. https://doi.org/10.1002/app.11050

Tamura, I.; Kagota, K.I.; Yasuda, Y.; Yoneda, S.; Morita, J.; Nakada, N.; Kameda, Y.; Kimura K.; Tatarazako, N.; Yamamoto, H. 2012. Ecotoxicity and screening level ecotoxicological risk assessment of five antimicrobial agents: triclosan, triclocarban, resorcinol, phenoxyethanol and p-thymol. $J$ Appl Toxicol 33(11): 1222-1229. https://doi.org/10.1002/jat.2771

Thomas, M.G.; Abraham, E.; Jyotishkumar, P.; Maria, H.J.; Pothen, L.A.; Thomas, S. 2015. Nanocelluloses from jute fibers and their nanocomposites with natural rubber: Preparation and characterization. Int J Biol Macromol 81: 768-777. https://doi.org/10.1016/j.ijbiomac.2015.08.053

Tian, G.; Zhuang, J.; Fu, Y.; Wang, Z.; Li, Q. 2019. Enhanced mechanical strength of polyethylene-based lignocellulosic-plastic composites by cellulose fibers. BioResources 14(1): 1668-1678. https://ojs.cnr.ncsu. edu/index.php/BioRes/article/viewFile/BioRes_14_1_1668_Tian_Enhanced_Mechanical_Strength_Polyethylene/6610

Varghese, S.; Kuriakose, B.; Thomas, S. 1994. Stress relaxation in short sisal-fiber-reinforced natural rubber composites. J Appl Polym Sci 53(8): 1051-1060. https://doi.org/10.1002/app.1994.070530807

Wang, J.; Wu, W.; Wang, W.; Zhang, J. 2011. Effect of a coupling agent on the properties of hemp-hurdpowder-filled styrene-butadiene rubber. J Appl Polym Sci 121(2): 681-689. https://doi.org/10.1002/app.33744

Wisittanawat, U.; Thanawan, S.; Amornsakchai, T. 2014. Mechanical properties of highly aligned short pineapple leaf fiber reinforced - Nitrile rubber composite: Effect of fiber content and Bonding Agent. Polym Test 35: 20-27. https://doi.org/10.1016/j.polymertesting.2014.02.003

Wisojodharmo, L.A.; Arti, D.K.; Pravitasari, R.D.; Hidayat, A.S.; Nisa, A.I.; Ariesta, M.N. 2020. Effect of Tackifier Addition on Cushion Compound Formulation for Tire Retreading Application. Indones $J$ App Chem 21(2): 81-90. https://doi.org/10.14203/jkti.v21i2.427

Zhang, B.; Gu, B.; Yu, X. 2015. Failure behavior of resorcinol-formaldehyde latex coated aramid short-fiber-reinforced rubber sealing under transverse tension. J Appl Polym Sci 132(12): 1-8. https://doi.org/10.1002/ app. 41672

Zhang, X.X.; Lu, C.H.; Liang, M. 2007. Preparation of rubber composites from ground tire rubber reinforced with waste-tire fiber through mechanical milling. J Appl Polym Sci 103(6): 4087-4094. https://doi. org/10.1002/app.25510

Zhou, Y.; Fan, M.; Chen, L.; Zhuang, J. 2015. Lignocellulosic fibre mediated rubber composites: An overview. Compos B Eng 76: 180-191.https://doi.org/10.1016/j.compositesb.2015.02.028 